\title{
A hypomorphic mouse model of dystrophic epidermolysis bullosa reveals mechanisms of disease and response to fibroblast therapy
}

\author{
Anja Fritsch, ${ }^{1}$ Stefan Loeckermann, ${ }^{1}$ Johannes S. Kern, ${ }^{1,2}$ Attila Braun, ${ }^{3}$ Michael R. Bösl, ${ }^{3}$ \\ Thorsten A. Bley, ${ }^{4}$ Hauke Schumann, ${ }^{1}$ Dominik von Elverfeldt, ${ }^{5}$ Dominik Paul, ${ }^{5}$ Miriam Erlacher, ${ }^{6}$ \\ Dirk Berens von Rautenfeld, ${ }^{7}$ Ingrid Hausser, ${ }^{8}$ Reinhard Fässler, ${ }^{3}$ and Leena Bruckner-Tuderman ${ }^{1}$

\begin{abstract}
${ }^{1}$ Department of Dermatology, University Medical Center Freiburg, Freiburg, Germany. ${ }^{2}$ Faculty of Biology, University of Freiburg, Freiburg, Germany. ${ }^{3}$ Department of Molecular Medicine, Max Planck Institute of Biochemistry, Martinsried, Germany. ${ }^{4}$ Department of Radiology,

${ }^{5}$ Department of Diagnostic Radiology and Medical Physics, and ${ }^{6}$ Department of Pediatrics, University Medical Center Freiburg, Freiburg, Germany ${ }^{7}$ Department of Functional and Applied Anatomy, Hannover Medical School, Hannover, Germany. ${ }^{8}$ Department of Dermatology,
\end{abstract} \\ University of Heidelberg, Heidelberg, Germany.
}

\begin{abstract}
Dystrophic epidermolysis bullosa (DEB) is a severe skin fragility disorder associated with trauma-induced blistering, progressive soft tissue scarring, and increased risk of skin cancer. DEB is caused by mutations in type VII collagen. In this study, we describe the generation of a collagen VII hypomorphic mouse that serves as an immunocompetent animal model for DEB. These mice expressed collagen VII at about $10 \%$ of normal levels, and their phenotype closely resembled characteristics of severe human DEB, including mucocutaneous blistering, nail dystrophy, and mitten deformities of the extremities. The oral blistering experienced by these mice resulted in growth retardation, and repeated blistering led to excessive induction of tissue repair, causing TGF- $\beta 1$-mediated contractile fibrosis generated by myofibroblasts and pseudosyndactyly in the extremities. Intradermal injection of WT fibroblasts resulted in neodeposition of collagen VII and functional restoration of the dermal-epidermal junction. Treated areas were also resistant to induced frictional stress. In contrast, untreated areas of the same mouse showed dermal-epidermal separation following induced stress. These data demonstrate that fibroblast-based treatment can be used to treat DEB in a mouse model and suggest that this approach may be effective in the development of clinical therapeutic regimens for patients with DEB.
\end{abstract}

\section{Introduction}

Skin integrity and resistance to mechanical stress rely on the function of the dermal-epidermal junction zone (DEJZ), which anchors the epidermis to the underlying dermal matrix. The supramolecular cell adhesion complexes at the DEJZ mediate interactions of the cytoskeleton in basal keratinocytes with the basement membrane and the extracellular anchoring fibrils, which emanate from the basement membrane into the dermis and entrap dermal collagen bundles, thus establishing stable dermal-epidermal cohesion (1).

The main component of the anchoring fibrils is collagen VII, a homotrimeric collagen synthesized by keratinocytes and fibroblasts (2). Fibril formation and deposition at the DEJZ requires proteolytic processing of procollagen VII to mature collagen (3). Loss of collagen VII functions in dystrophic epidermolysis bullosa (DEB) leads to absence or anomalies of the anchoring fibrils and to dermal-epidermal tissue separation. DEB refers to a clinically heterogeneous group of disorders including recessively and dominantly inherited subtypes $(4,5)$. All forms of DEB are allelic and caused by mutations in the collagen VII gene, COL7A1. Early investigations demonstrated reduced amounts of anchoring fibrils and collagen VII in the skin of patients with mild and moderate, reces-

Nonstandard abbreviations used: CTGF, connective tissue growth factor; DEB, dystrophic epidermolysis bullosa; DEJZ, dermal-epidermal junction zone; PGK-Neo, phosphoglycerate kinase promoter-driven neomycin phosphotransferase expression (cassette); RDEB, recessive DEB.

Conflict of interest: The authors have declared that no conflict of interest exists. Citation for this article: J. Clin. Invest. 118:1669-1679 (2008). doi:10.1172/JCI34292. sive and dominant DEB and their complete absence in severe recessive DEB (RDEB) $(1,5)$. However, despite worldwide disclosure of more than 300 distinct COL7A1 mutations, their different homozygous, heterozygous, and compound heterozygous combinations $(6,7)$, and a broad spectrum of biological and clinical phenotypes, the precise genotype-phenotype correlations and molecular disease mechanisms in DEB remain to be elucidated.

RDEB is a stigmatizing and cancer-prone skin condition for which no curative therapy exists at present. The clinical phenotype is characterized by skin blistering with mucosal involvement, soft tissue scarring, alopecia, nail dystrophy, and/or development of mitten deformities of the hands and feet. The spectrum extends from localized mild involvement of trauma-exposed sites to severe generalized disease, which has a high risk of skin cancer. Most patients with severe RDEB develop squamous cell carcinoma of the skin by the age of 40 years (5). About two-thirds of affected individuals develop partial or total mitten deformities of the hands and feet. It is intriguing that the mitten deformities represent predilection sites for squamous cell carcinoma, especially in light of reports that abnormal stroma can modulate the oncogenic potential of adjacent epithelia (8). Therefore, elucidation of the molecular mechanisms underlying progressive scarring in DEB is urgently needed as a prerequisite for the development of biologically valid therapies for skin fragility, scarring, and cancer in DEB.

To address these questions, it is necessary to have a viable animal model that mimics DEB at both genetic and phenotypic levels. In this study, we generated a hypomorphic mouse that fulfilled these criteria. Previously, targeted inactivation of the Col7al gene generat- 
A

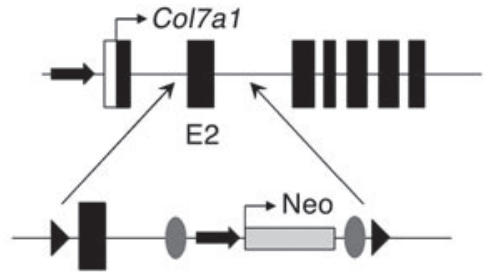

C Col7a1wiwt
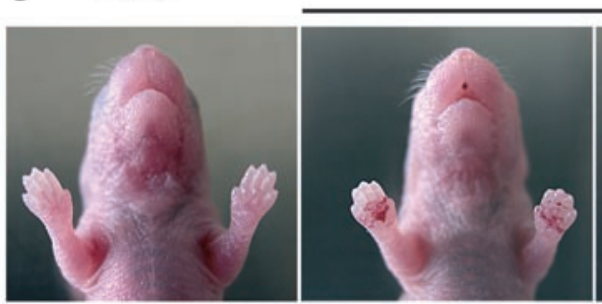

B

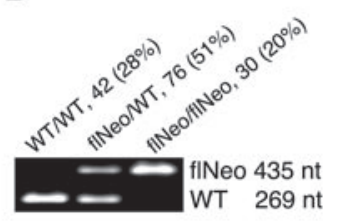

Col7a $1^{\text {IINeofINeo }}$

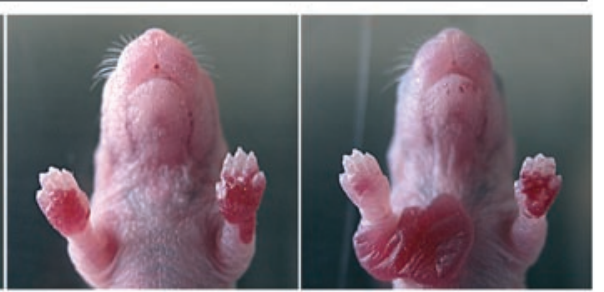

Figure 1

Homozygous Col7a $1^{\text {fINeo }}$ mice develop blisters early after birth. (A) An 11-kb genomic fragment of Col7a1 was replaced by the targeting construct, flanking exon 2 (E2) with 2 loxP sites (black triangles). Additionally, the Neo cassette (gray box) was introduced downstream of exon 2, with 2 Frt sites (gray ovals) allowing Flp-mediated removal of the cassette. Right-facing thick black arrows indicate

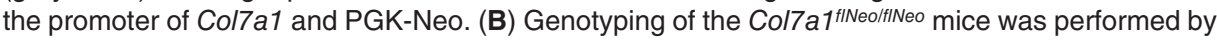
PCR detecting the presence of the $5^{\prime}$ loxP site, with amplicons of $269 \mathrm{nt}$ for the WT allele and $435 \mathrm{nt}$ for the transgenic allele. The percentages of the genotypes generated are in accordance with normal Mendelian inheritance $\left(\chi^{2}=2.12 ; P=0.34\right)$. (C) Hemorrhagic blisters were visible within $24 \mathrm{~h}$ of birth in the paws of Col7at $1^{\text {fINeolfINeo mice. }}$

ed a severely affected collagen VII knockout mouse, which was born with extensive cutaneous blistering and died during the first 2 weeks of life due to complications arising from the blistering (9). Therefore, the knockout was not very useful for experiments or therapeutic regimens requiring long-term monitoring. In the present study we developed a collagen VII hypomorphic mouse, which had 10\% of the normal collagen VII levels in the skin and developed all the symptoms of severe RDEB. Its survival to adulthood and immunocompetence makes it ideally suited for analysis of molecular pathogenesis of DEB and evaluation of novel therapeutic strategies.

\section{Results}

Homozygous Col7 a $1^{\text {fNeo }}$ mice develop blisters after birth. To generate a mouse model for conditional inactivation of collagen VII expression, a targeting construct containing a phosphoglycerate kinase promoter-driven neomycin phosphotransferase expression cassette (PGK-Neo cassette) and 2 flanking Frt sites that allow its Flp-mediated removal (Figure 1A) were introduced into intron 2 of Col7a1. Col7a $1^{W T / W T}$, Col7 a $1^{\text {flNeo/WT }}$, and Col7a $1^{\text {flNeofflNeo animals }}$ were born in a normal Mendelian ratio (Figure 1B) and were indistinguishable at birth. However, within 24-48 hours, Col7 a fliNeofflNeo mice developed hemorrhagic blisters on the soles of fore and hind paws and, occasionally, also on the ears and mouth. The extent of blister formation varied between individual animals, ranging from small blisters on the fore paws to extensive blistering on the extremities and the trunk (Figure 1C).

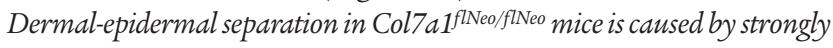
reduced collagen VII and anchoring fibrils. Histopathology of the skin

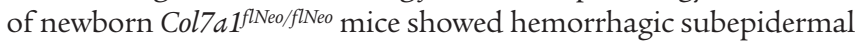
blisters (Figure 2A). Immunofluorescence staining with antibodies to collagen VII revealed weak reactivity in the skin of Col7 a 1 f fiNeofflNeo

fibrils is formed and provides some stability to the DEJZ.

Aberrant splicing of collagen VII mRNA leads to reduced expression levels in Col7 a $1^{\text {flNeofffNeo }}$ mice. Introduction of the Col7 a f f ${ }^{\text {fNeo }}$ allele obviously interfered with normal collagen VII expression. Negative effects of intronic insertions of PGK-Neo cassettes on gene expression have been observed previously (10-12). The mechanisms of inhibition include transcriptional downregulation of the target gene $(10,11)$ as well as alterations in the splicing pattern (12). To characterize collagen VII expression from the Col7a $1^{\text {flNeo }}$ allele in more detail, collagen VII mRNA from immortalized Col7a $1^{\text {WT } W T}$, Col7a $1^{\text {INEo/WT }}$, and Col7 a f flNeofflNeo keratinocytes was amplified with primers annealing in Col7a1 exons 1 and 2. This showed no difference in the amount and length of this part of collagen VII mRNA, which suggested that transcription of Col7a1 was not impaired and splicing of exon 1exon 2 was normal. However, amplification of exon 2-exon 3 revealed several aberrant transcripts in Col7 a flNeofflNeo mice. RT-PCR with primers annealing in exon 2 and in the PGK-Neo cassette demonstrated transcripts harboring Neo sequences in Col7a f flNeofflNeo and Col7 a f flNoo/WT mice (Figure 3A). Rapid amplification of cDNA $3^{\prime}$-ends (3'-RACE) confirmed that part of the collagen VII mRNAs stopped before exon 3 , terminated by the polyadenylation signal of the PGKNeo cassette. The main transcript variants in Col7 a $1^{\text {flNeofflNeo }}$ keratinocytes are shown in Figure 3B. Evidently, only the correct splicing product can be translated into collagen VII protein. Densitometry revealed about $75 \%$ of WT levels in Col7 a 1 f flNeo/WT mice and about $9 \%$

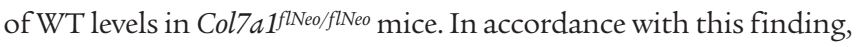
the amount of collagen VII protein in newborn Col7 a f flNeoffINeo skin and keratinocytes was reduced to about 10\% of WT levels (Figure 3C). Cultured fibroblasts synthesized about $50 \%$ of their normal levels, which are significantly lower than in keratinocytes (2) and are not sufficient for adequate dermal-epidermal adherence. 

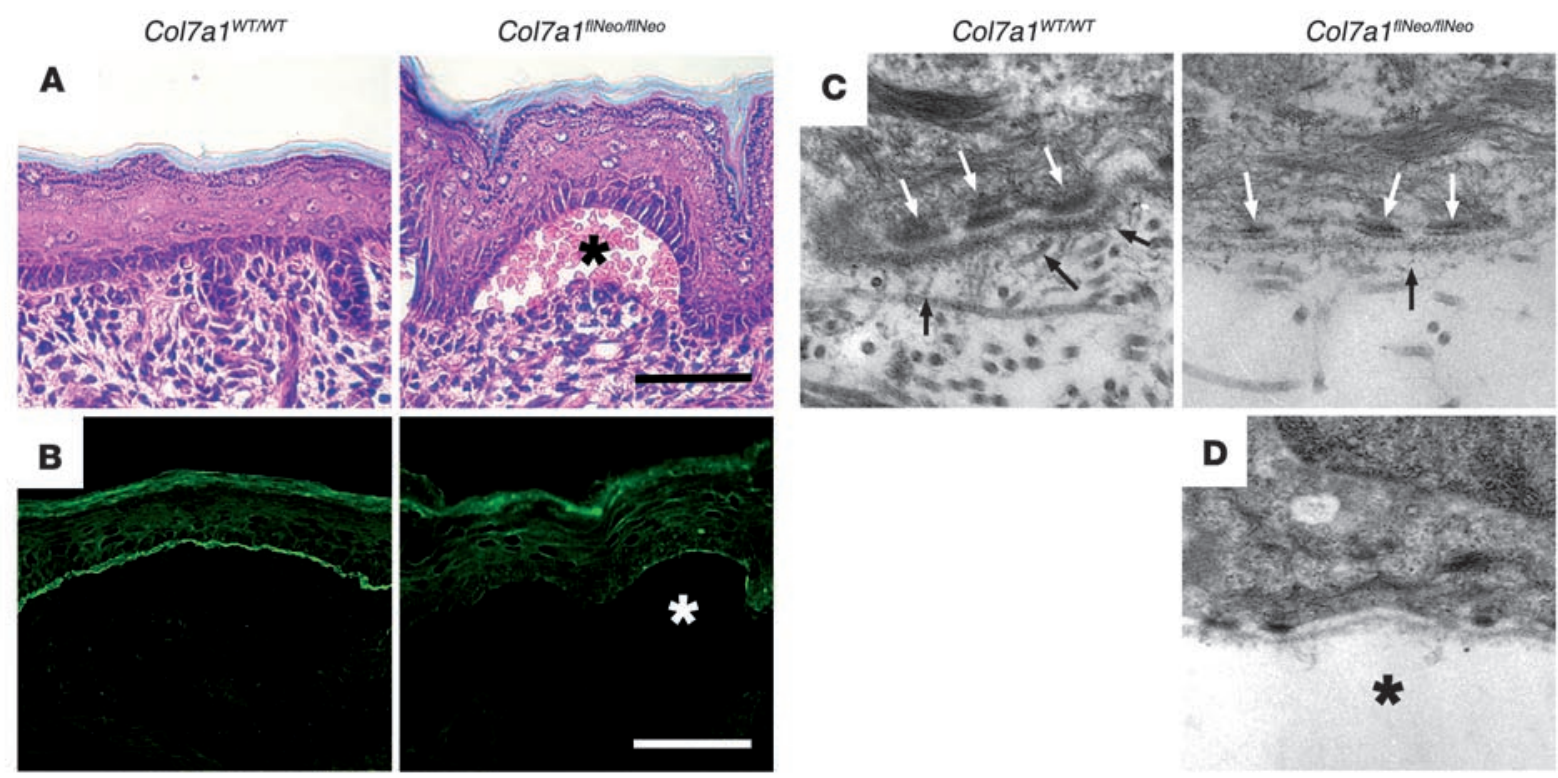

Figure 2

Dermal-epidermal separation in the Col7a1 $1^{\text {fINeo/fINeo }}$ mouse is caused by strongly reduced collagen VII deposition. (A) Histological analysis (H\&E

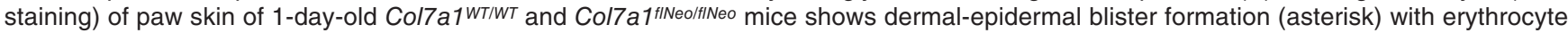

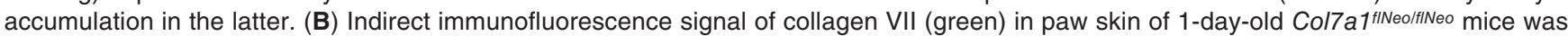
strongly reduced compared with Col7a1WT/WT mice. Asterisk indicates the blister cavity. Scale bars: $50 \mu \mathrm{m}$. (C and D) Ultrastructural analysis

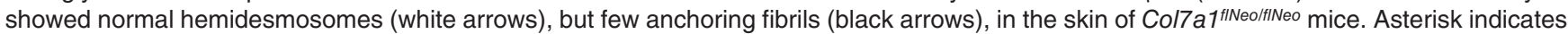
the blister cavity. Original magnification, $\times 195,000$.

Malnutrition causes growth retardation and reduced life expectancy in Col7a $1^{\text {flNeofflNeo }}$ mice. The Col7a $1^{\text {flNeo/flNeo }}$ mice appeared normal at birth. However, postnatal weight gain was slower than in the littermates, although the overall development - including the opening of the eyes and the start of first hair cycle - was not altered. At 2-3 weeks of age, these mice were significantly smaller, their average weight half that of Col7a $1^{W T / W T}$ and Col7a $1^{\text {flNoo/WT }}$ littermates. This difference was maintained until adolescence (Figure 4A). Overall, the general condition of Col7a $1^{\text {flNeo/flNeo }}$ mice - as judged by weight, posture, activity, and fur texture - was reduced. Of the Col7 a fllNeofflNeo mice, $67 \%$ died before weaning at day 28 (Table 1). Histopathology of the tongue showed blistering (Figure 4B), which was also macroscopically visible during necropsy and causes severe malnutrition. An accumulation of gases in the stomach and in the lower digestive tract was evident, but the microbiology of the intestinal flora was normal. Anemia, a common symptom in DEB, was not found

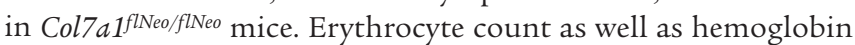
and hematocrit levels were within the normal range for mice and did not differ from those of Col7 $11^{W T / W T}$ animals.

The high mortality during the first 4 postnatal weeks and the poor general condition reflected the inability of Col7a $1^{\text {flNeo flNeo mice }}$ to adapt to solid food, because change to a liquid diet consisting of infant milk cereal with a dietary supplement starting from postnatal day 10 allowed them to gain weight and survive longer than 100 days. Because most Col7a $1^{\text {flNeo/flNeo }}$ mice in good general condition were used for experiments, the precise percentage of the animals surviving more than 100 days cannot be given. At termination of the present experiments, 5 animals lived more than 100 days, the oldest surviving 173 days. Gender does not appear to be a survival factor: of the mice that survived $28-100$ days, $54 \%$ were male and $46 \%$ female (Table 1). In conclusion, the presence of $10 \%$ of normal levels of collagen VII in the skin and mucous membranes confers sufficient stability to the DEJZ for long-term survival.

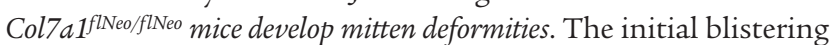
in the paws of the Col7a1 flNeo/flNeo mice healed with scarring (Figure 5 , D-G). Beginning at 2-3 weeks of age, the condition slowly worsened to include nail dystrophy and shortened or missing digits (Figure 5E). By 9-12 weeks, partial or complete mitten deformities were observed (Figure 5, F and G). The extent of these changes reflected cumulative exposure to shearing forces and trauma; notably, the fore paws were more severely affected than the hind paws. The time course and the macroscopic appearance of the deformities closely resembled the lesions in patients with severe RDEB (Figure 5, A-C), most of which develop mitten hands and feet by $6-8$ years of age. Thus, the collagen VII hypomorphic mouse is ideally suitable to analyze the mechanisms underlying the deformities in DEB.

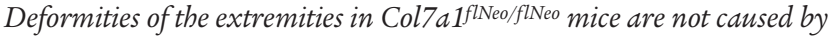
bone defects and represent pseudosyndactyly. High-resolution digital radiography (Figure 6, A-D) and corresponding high-resolution MRI (Figure 6, E-G) of Col7a $1^{W T / W T}$ and Col7 a $1^{\text {flNeofflNeo mice in sag- }}$ ittal, transversal, and coronal orientations revealed that the various stages of mutilating deformities were not caused by bone defects, but rather by soft tissue accumulation and contraction. The mineral content in the long bones of the extremities was reduced in the Col7a1 flNoo/flNeo mice (Figure 6, B and C). The severe contractures and reduced bone mineral content of Col7a $1^{\text {flNeo/flNeo }}$ animals closely resembled the radiographic images of mitten deformities and osteopenia in patients with severe RDEB (Figure 6D).

Pseudosyndactyly is a consequence of aberrant contractile fibrosis. After ruling out bone anomalies as cause for the mitten deformities in the Col7a1 $1^{\text {flNoo/flNeo }}$ mice, we predicted that dysregulated wound healing underlies the phenotype. Histopathological examination 

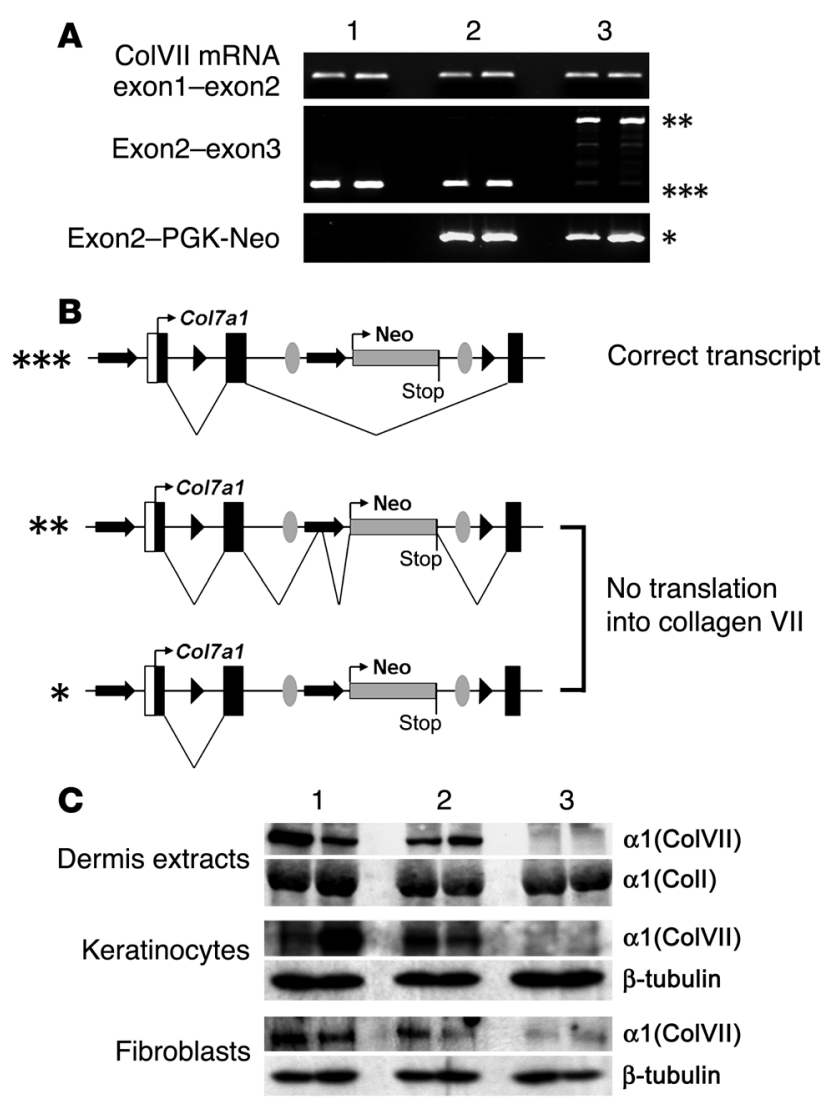

of the paws of 80-day-old Col7a f flNeofflNeo mice revealed dermal-epidermal separation, reactive thickening of the epidermis, accumulation of connective tissue, and markedly decreased elastic fibers in the dermis (Figure 7, A and B), indicating strong dermal fibrosis. Immunofluorescence staining with the CD11b antibody that predominantly recognizes granulocytes and macrophages revealed the presence of inflammatory cells in the upper dermis (Figure $7 \mathrm{C}$ ), which suggested that these had not dispersed from the wound site in a normal manner (13). As a consequence, TGF- $\beta$ and connective tissue growth factor (CTGF) accumulated in the dermis of Col7 a f flNeofflNeo mice (Figure 7, D and E). TGF- $\beta$ is the main myofibroblast inducer, and the dermis consistently showed a high number of $\alpha$-SMA-positive myofibroblasts (Figure 7F), which exert clearly stronger contractile activity than do $\alpha$-SMA-negative fibroblasts (14). This was accompanied by strong upregulation of tenascin C (Figure 7G), a marker for ongoing inflammatory response and fibrotic changes in the dermal matrix (15). Taken together, these data indicate that the pseudosyndactyly and severely impaired organ functions of the extremities in Col7 a $1^{\text {flNeoffINeo }}$ mice, and presumably in humans, result from aberrant tissue repair and myofibroblast-mediated excessive contraction and extracellular matrix deposition.

Removal of the PGK-Neo cassette rescues the phenotype of Col7 a f flineofflineo mice. If all the above-described symptoms were a direct consequence of reduced collagen VII levels in the skin and mucous membranes, removal of the PGKNeo cassette should rescue the phenotype. Indeed, breeding the Col7 a $1^{f I N e o}$ strain to a Flp-expressing strain, which

\section{Figure 3}

Aberrant mRNA splicing causes reduced expression of collagen VII

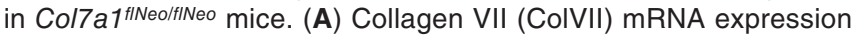
was analyzed in immortalized keratinocytes of Col7a1WT/WT (lanes 1),

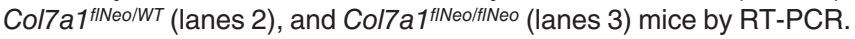
Amplification of exon 1-exon 2 showed no difference in expression levels between the genotypes. In contrast, amplification of exon 2exon 3 revealed aberrant splice variants in Col7a $1^{f I N e o / f I N e o}$ keratinocytes. Asterisks indicate the normal splice product $\left.{ }^{* * *}\right)$ and the longest aberrant product $\left({ }^{* *}\right)$. A transcript variant containing part of the PGK-

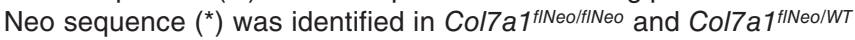
cells. (B) Schematic representation of the major transcript variants, the majority of which cannot be translated into protein. The asterisks denote the variants shown in A. Black boxes indicate Col7a1 exons; gray boxes indicate the Neo cassette; bold black arrows indicate the promoter PGK; "Stop" indicates the translational stop of neomycin phosphotransferase; triangles indicate loxP sites; ovals indicate Frt sites. (C) Collagen VII protein levels were analyzed by immunoblotting extracts of dermis, immortalized keratinocytes, and fibroblasts of newborn Col7a $1^{\text {WT/WT }}$ (lanes 1), Col7a $1^{\text {flNeo/WT }}$ (lanes 2), and Col7a $1^{\text {flNeol/IINeo }}$ (lanes 3) mice. Each individual lane contains samples of an independent animal. Collagen VII was detected with the NC2-10 antibody (44). As loading controls, the Coomassie blue-stained band of $\alpha 1$ chain of collagen I (Coll) or immunoblotting with anti- $\beta$-tubulin were used.

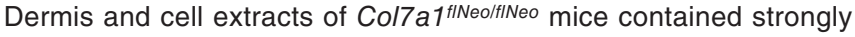
reduced amounts of collagen VII. leads to removal of the PGK-Neo expression cassette, restored normal expression of collagen VII even in the presence of the remaining loxP sites (Figure 8A). We bred C57BL/6-TgH(Col7a1flNeo)288LBT mice to B6;SJL-Tg(ACTFLPe)9205Dym/J mice and subsequently inbred the offspring to remove the Flp transgene. The resulting Col7 a 1fl strain [C57BL/6-TgH(Col7a1fl)288LBT] generated phenotypically normal Col7a $1^{W T / W T}$, Col7a $1^{f l / W T}$, and Col7a $1^{f l / f l}$ offspring in a normal Mendelian ratio; these mice had a normal body weight, lived to adulthood, and were fertile (Figure 8B). There was no difference in collagen VII expression among Col7a $1^{W T / W T}, \mathrm{Col7a} 1^{f / W T}$, and Col7 a 1/flfl mice (Figure 8, C and D).

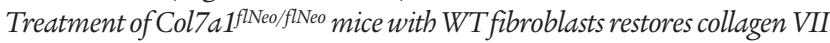
deposition and function at the DEJZ. To test fibroblasts as a source of ther-
Table 1

Survival of Col7a $1^{\text {fiNeoffineo mice }}$

Total

Spontaneous death before $28 \mathrm{~d}$

Sacrificed after $28 \mathrm{~d}$, poor general condition $28-100 d$

$>100 \mathrm{~d}$

Sacrificed after $28 \mathrm{~d}$, experiments $28-100 \mathrm{~d}$ $>100 \mathrm{~d}$

\section{$\%$}

118

67

$10(5 \mathrm{M}, 5 \mathrm{~F})$

$9(4 \mathrm{M}, 5 \mathrm{~F})$

$1(1 \mathrm{M}, 0 \mathrm{~F})$

$29(16 \mathrm{M}, 13 \mathrm{~F})$

25 (13 M, $12 \mathrm{~F})$

$4(3 \mathrm{M}, 1 \mathrm{~F})$

Newborn mice were fed as described in Methods. ASex was not determined for mice in this group. 


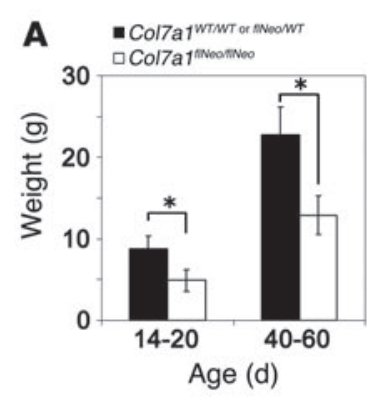

B

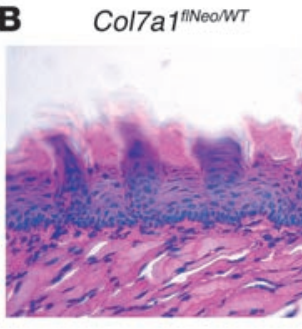

Col7a1 $1^{\text {IINeorineo }}$

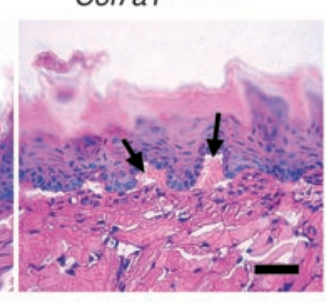

Figure 4

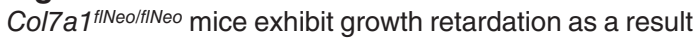
of malnutrition. Although normal at birth, Col7a $1^{\text {fINeolfINeo }}$ mice grew slower than their Col7a1WT/WT or Col7a $1_{\text {fINeo/WT }}$ littermates. (A) Mean $\pm \mathrm{SD}$ weight of Col7a $1^{\text {fINeo/fINeo }}$ $(n=25[14-20 \mathrm{~d}] ; 13[40-60 \mathrm{~d}])$ compared with control littermates $(n=50)$. ${ }^{*} P<0.001$, Student's $t$ test. (B) H\&E staining of tongue of 17-day-old mice, with arrows highlighting hemorrhagic blisters. Scale bar: $50 \mu \mathrm{m}$.

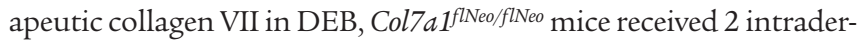
mal injections of $20 \times 10^{6}$ fibroblasts into a defined area on the dorsal skin, with an interval of 7 days between injections. Of the 19 mice injected, 16 received WT fibroblasts, and 3 received Col7a $1^{\text {flNeooflNeo }}$ fibroblasts as a control. At 7, 14, and 21 days after the second injection, the treated and untreated skin areas were subjected to a functional stress test, excised, and analyzed with morphological and biochemical methods. Intriguingly, the treatment with WT fibroblasts resulted in both deposition of collagen VII at the DEJZ and functional restoration. At 7 days after the second injection, collagen VII at the DEJZ was clearly increased compared with untreated skin (Figure 9, A and B). The increased amounts persisted throughout the entire 21-day observation period (Figure 9C). Semiquantitative comparison of relative mean collagen VII immunofluorescence intensities at the DEJZ ( $n=5$ per group) was performed with a confocal microscope using identical image settings. We found a statistically significant increase of the signal intensity 7 days after fibroblast treatment compared with untreated skin $(P<0.01$; Figure 9E). The increase was about 3.5-fold, and the collagen VII levels in treated skin were estimated to correspond to about $25 \%-30 \%$ of WT levels. In line with this, collagen VII mRNA expression, as analyzed by RT-PCR amplification of Col7a1 exon 1-exon 3 of total skin RNA, was strongly increased 7 days after treatment compared with untreated controls (Figure 9F). In occasional sections, an inflammatory cell infiltrate was observed in the dermis, but neither direct nor indirect immunofluorescence staining showed evidence of an antibody response to components of the DEJZ 3 weeks after treatment (data not shown). Injection of an equal amount of Col7a1 flNeo/flNeo fibroblasts as a control caused a slight deposition of collagen VII at the DEJZ (data not shown). Microblisters, which were regularly present in untreated skin of the hypomorph, were absent in the skin treated with WT fibroblast injections. Even intense mechanical stress (i.e., shearing forces applied by repeated unidirectional rubbing with a pencil eraser) did not induce dermal-epidermal separation and blisters (Figure 9, G and H), indicating that the therapeutically supplemented collagen VII restored the stability of the skin.

\section{Discussion}

Through generation of the collagen VII hypomorphic mouse, we established what we believe to be the first nonlethal, immunocompetent mouse model for DEB. The advantage of this model is that the pathology is caused entirely by quantitative reduction of normal collagen VII in the skin, and not by interference of mutated molecules, which would generate a variety of abnormal structures and complex biological responses. In view of therapeutic considerations it is notable that the presence of one-tenth of normal full-length collagen VII in the skin of the hypomorphic mouse conferred a substantially milder phenotype and better prognosis than the complete Col7a1 knock- out, which is perinatally lethal (9). Instead of birth trauma-induced blistering involving the entire integument of the knockout mouse, the hypomorphic mice developed localized blisters in the paws and, with time, disseminated blistering and scarring. Severe erosions of skin and oral mucosa led to demise of the null mice within the first 2 weeks of life (9), but the Col7a $1^{\text {flNeo/flNeo }}$ mice survived to adulthood and can have a long life if kept on a liquid diet.

As in humans with COL7A1 mutations, skin fragility, nail dystrophy, digit malformations, pseudosyndactyly, and growth retardation were clinical hallmarks of adult Col7a $1^{\text {flNeo/flNeo }}$ mice. Even the time course of pseudosyndactyly development was comparable in mice and humans: children with severe DEB develop mitten deformities by the age of 6-8 years, and these deformities were seen in

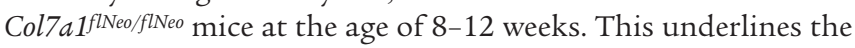
suitability of this mouse as a model for dissecting disease mechanisms and testing molecular therapies for DEB. The only symptom not present in these mice was anemia, which suggests that in patients this is secondary to chronic inflammation, bleeding, malnutrition, and other factors influencing erythropoiesis. Development of squamous cell carcinoma, a severe complication of human DEB, was not seen in the mice as yet. However, the numbers and the age of the mice available may still be too low for long-term follow-up, because patients with RDEB usually do not develop

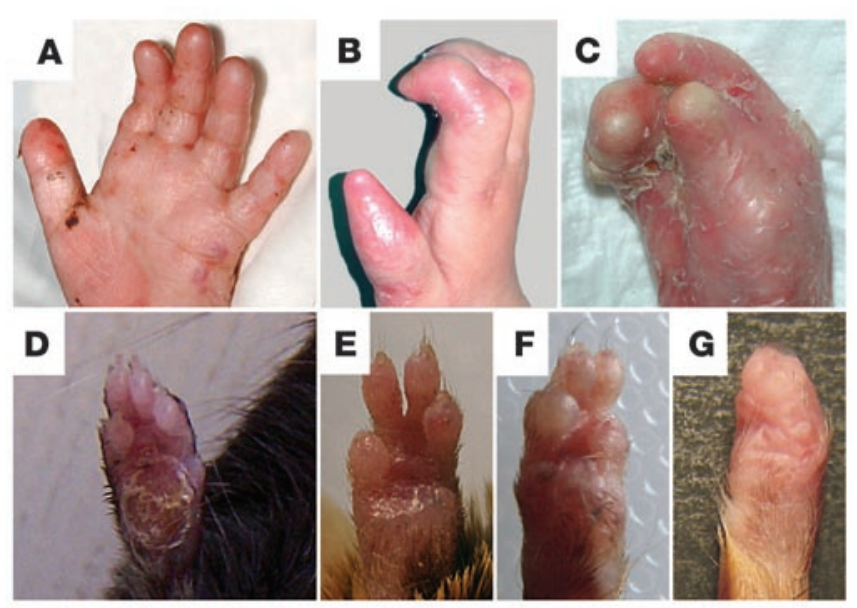

Figure 5

Mitten deformities in the extremities of the Col7a $1^{\text {fINeo/fINeo mice closely }}$ resemble severe human RDEB. (A-C) Hands in human RDEB patients at 2.5 years $(\mathbf{A}), 6.5$ years $(\mathbf{B})$, and 7.5 years of age $(\mathbf{C})$. (D-G) Fore

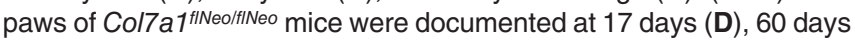
$(\mathbf{E})$, and 80 days of age ( $\mathbf{F}$ and $\mathbf{G})$. The clinical phenotypes in RDEB

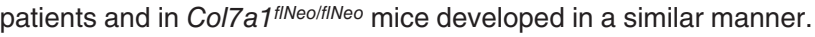



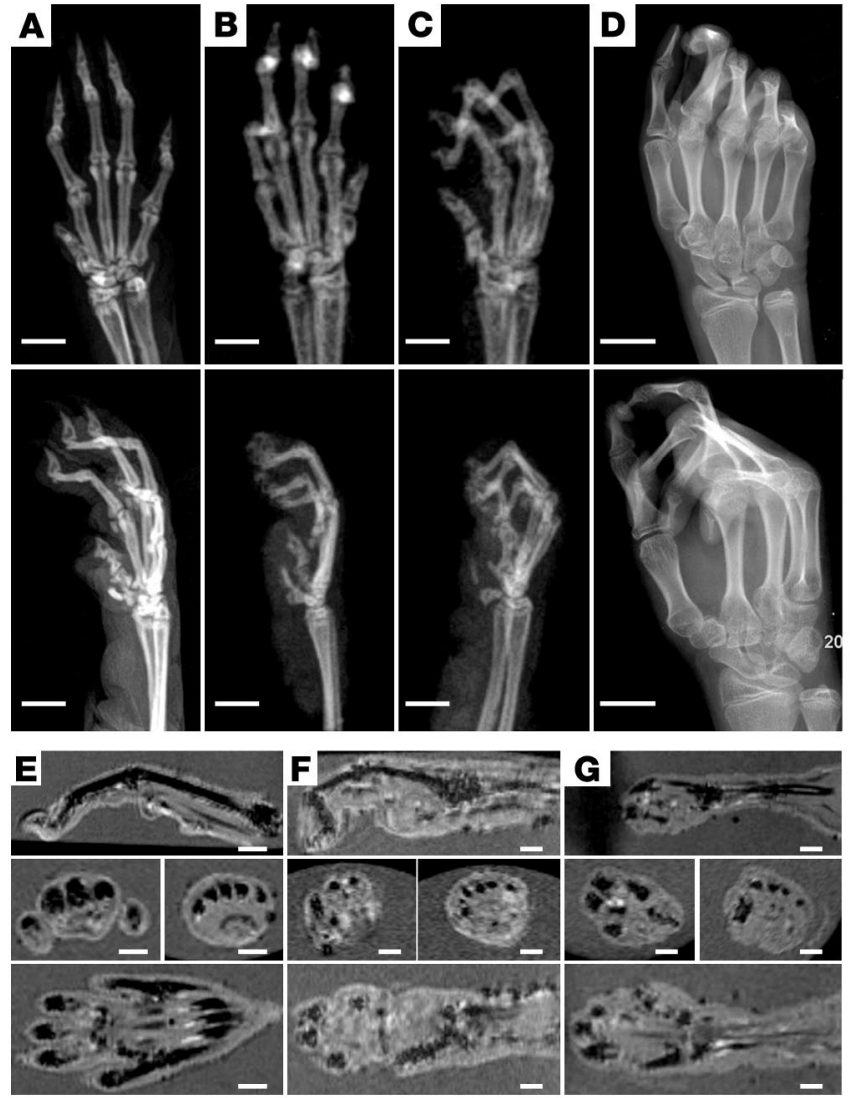

Figure 6

Mitten deformities of the extremities in the Col7a $1^{\text {fINeo/fINeo mouse are }}$ not primarily caused by bone abnormalities. (A-D) High-resolution digital radiography in 2 planes of the extremities of Col7a1WT/WT (A)

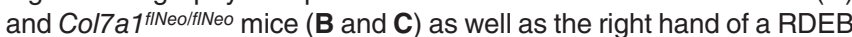
patient, demonstrating severe mitten deformity (D). Col7a $1^{\text {fINeo/fINeo }}$ mice are shown both mildly (B) and strongly (C) affected. Top panels, anterior-posterior; bottom panels, lateral. Note the reduced mineral content of the long bones in the extremities of the mice demonstrated by increased X-ray transparency. (E-G) Corresponding high-resolution MRI in sagittal (top panels), transversal (middle panels), and coronal (bottom panels) orientation in mildly $(\mathbf{F})$ and strongly affected

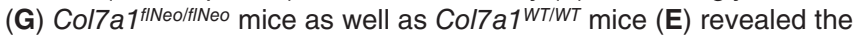
extent of deformities and thickening of the soft tissues. Scale bars: $1 \mathrm{~mm}$ (A-C and $\mathbf{E}-\mathbf{G}) ; 20 \mathrm{~mm}$ (D).

tumors until after the age of 20 years. It will be of great interest in the future to test the susceptibility of the mice to spontaneous and induced tumor development.

The mitten deformities of the hypomorphs resulted from a softtissue process: X-ray and MRI revealed the bony structures of the extremities remained intact, except for osteopenia. Pseudosyndactyly developed as a consequence of aberrant tissue repair and high contractile forces generated by myofibroblasts. During normal wound healing, a well-orchestrated cascade of cellular events takes place in the dermis. In the early phase, neutrophils are the predominant inflammatory cell type in the wound margins, preventing infection by killing microbes. Later, monocytes extravasate from surrounding vessels and differentiate into macrophages that remove matrix and necrotic/apoptotic cells, but after wound closure, the inflammatory cells disperse from the site of injury $(13,16$,
17). Simultaneously, fibroblasts differentiate into contractile and secretory myofibroblasts that contribute to wound contraction and remodeling in a controlled, temporary manner $(14,18)$. This is not the case in the hypomorph, in which repeated minor traumatization of the paws leads to aberrant excessive tissue repair. At the age of 12 weeks, significant infiltration of CD11b-positive macrophages persisted in the skin, accompanied by upregulation of TGF- $\beta$ and CTGF, neoexpression of $\alpha$-SMA, excessive fibroblastto-myofibroblast differentiation, and induction of tenascin $\mathrm{C}$. These molecules and cells are key players in fibrotic processes with excessive contractile force and deposition of extracellular matrix $(14,19)$. Tenascin $C$ is not expressed in normal dermis, but is persistently upregulated in fibrotic disease (15). TGF- $\beta$ is considered the major growth factor promoting myofibroblast differentiation by inducing expression of $\alpha$-SMA. The presence of $\alpha$-SMA in stress fibers confers significantly stronger contractile activity to differentiated myofibroblasts than to control fibroblasts. In fact, $\alpha$-SMA can be regarded as a mechanosensory protein, because its expression and incorporation into preexisting actin stress fibers in myofibroblasts is enhanced when greater stress is generated by the microenvironment (14). Based on the above data $(14,15,19)$ and the present findings on mice with mitten deformities, we conclude that in DEB these processes cause a vicious cycle of enhanced contractility and matrix synthesis by myofibroblasts, progressive stiffness and rigidity of the connective tissue, greater stress, further stimulation of fibroblast-to-myofibroblast differentiation, secretion of matrix molecules, and so on, finally resulting in extensive pseudosyndactyly and severe impairment of tissue functions.

Understanding the above cellular and molecular mechanisms is of eminent importance for several reasons. First, DEB-associated squamous cell carcinoma, a lethal complication of the disease, occurs preferably at the sites of strong dermal fibrosis in the extremities. Organ homeostasis depends on undisturbed mesenchymal-epithelial interactions, and changes in the stromal connective tissue and/or chronic inflammation can induce pathological reactions of the epithelia, including cancer development $(20,21)$. In addition, chronic tissue damage can introduce DNA damage and thereby promote tumorigenesis $(22,23)$, and stromal cells can have a significant impact on the carcinogenic process in adjacent epithelia via paracrine TGF- $\beta$ signaling (8). Conversely, normal fibroblasts have been shown to convert malignant epithelia to morphologically benign lesions $(24,25)$. These observations suggest that correction of the chronic tissue damage, inflammation, and matrix and fibroblast functions in DEB could also have a significant positive impact as cancer prevention measures. Preliminary experiments with human skin from hands with mitten deformities indicate that the same mechanisms are active in the mutilating processes in DEB as in mouse tissues (A. Fritsch, unpublished observations). Therefore, the hypomorphic mouse will be an ideal animal model to investigate tumor development and susceptibility to tumor promotion.

Second, knowledge of the molecular events that cause aberrant tissue repair and high contractile forces in DEB may disclose novel targets for pharmacological intervention. For example, it is conceivable that antiinflammatory agents or modulators of TGF- $\beta, \alpha$-SMA, or tenascin $\mathrm{C}$ expression may become valuable in the prevention of the excessive fibrosis and consequent epithelial carcinogenesis in DEB.

Third, the therapeutic approach in this study - intradermal injection of normal fibroblasts - appears promising because these cells could restore the functions of both the DEJZ and the der- 


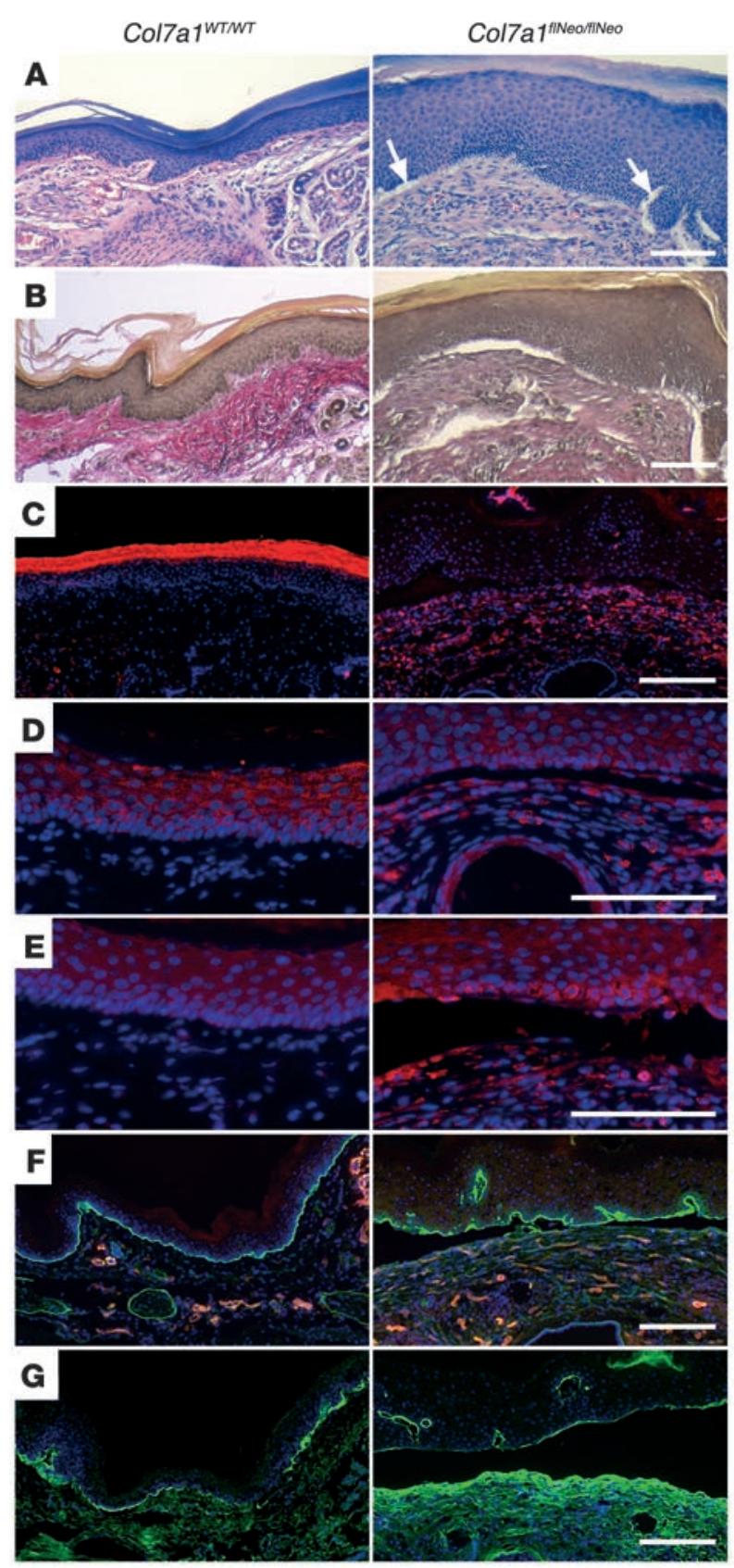

mal matrix. To date, different proof-of-principle experiments have demonstrated the feasibility to treat DEB by ex vivo gene therapy (26-28), cell-based therapy $(29,30)$, or injection of the protein itself (31), but all of these experiments have had significant drawbacks. Use of gene-corrected keratinocytes or fibroblasts overexpressing human collagen VII for skin reconstruction on athymic mice resulted in normal collagen VII deposition in the grafts (26-28). However, the transplants contained components of different species, and grafting of immunodeficient mice ignores the patients' putative immune response to the introduced protein. Antibodies to collagen VII can severely impair the function of anchoring fibrils, as evident in epidermolysis bullosa acquisita, an autoimmune blistering disease in humans and in a mouse model $(32,33)$. Dogs and sheep naturally afflicted with DEB represent immu-

\section{Figure 7}

Mitten deformities of the extremities result from excessive contractile fibrosis. (A) H\&E staining showed dermal-epidermal separation

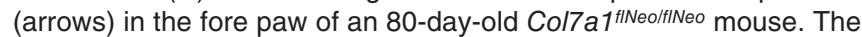
cellularity of the dermis was higher than that of Col7a1WT/WT mice. (B) van Gieson-elastica staining demonstrated distinct elastic fibers in Col7a1WT/WT mouse skin, whereas the elastic fibers were strongly

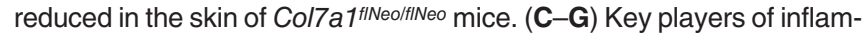
matory/fibrotic processes were assessed by immunofluorescence staining. Nuclei were stained with DAPI (blue). (C) Positive staining of CD11b, a marker for monocytes, dendritic cells, and macrophages, revealed the presence of inflammatory cells (red) in the Col7a $1^{\text {fINeolfiNeo }}$ dermis. (D) Expression of active TGF- $\beta 1$ (red) was enhanced in the

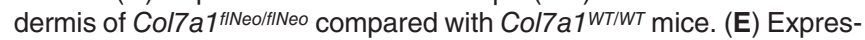
sion of CTGF (red) increased in both the dermis and the epidermis

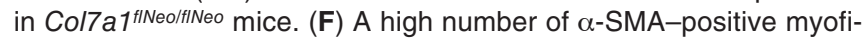
broblasts (red), which are not associated with vessels, were present

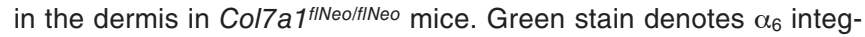
rin. (G) Tenascin C (green) was strongly upregulated in the dermis of

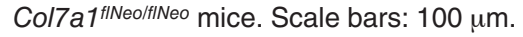

nocompetent animal models $(34,35)$, but difficulties in grafting and maintaining skin transplants in large animals have prevented them from being thoroughly examined. The only murine model, the complete knockout of collagen VII (9), presents with extensive skin blistering at birth, rendering therapeutic manipulation difficult. Furthermore, the mice die within the first 2 weeks of life, resulting in a narrow time frame for therapy induction and followup. Recently, a small pilot study reported injection of allogeneic fibroblasts into minute skin areas of DEB patients. Increased collagen VII was seen in epidermal keratinocytes and at the DEJZ, and this was interpreted as enhanced collagen VII synthesis and secretion by keratinocytes, stimulated by paracrine signals from the injected fibroblasts (36). Obviously, it will not be easy to carry out an extensive study in a human system to clarify the mechanisms.

Therefore, the Col7a $1^{\text {flNeo/flNeo }}$ mice offer a unique opportunity to analyze the efficacy and adverse effects of experimental treatments of DEB. Here we used intradermal injection of normal fibroblasts, $20 \times 10^{6}$ cells, for an area of $2 \times 3 \mathrm{~cm}$ on the back of a Col7a $1^{\text {flNeo/flNeo }}$ mouse, repeated after 1 week. At 7 days after the second injection, a significantly higher amount of collagen VII mRNA was present in treated skin. Deposition of collagen VII protein at the DEJZ was clearly increased and persisted for the entire observation period of 21 days. A confocal microscopy-based semiquantitative comparison of the intensity of collagen VII immunofluorescence signals showed a statistically significant increase of collagen VII immunofluorescence after fibroblast treatment. Remarkably, the treatment also restored the cohesive functions of the DEJZ. When the fibroblast-treated hypomorphic mice were exposed to a functional test using frictional rubber stress on days 7, 14, and 21 after injection, no blisters developed. In contrast, in untreated areas of the same mouse, the test induced microblisters. Another control, intradermal injection of the same number of fibroblasts

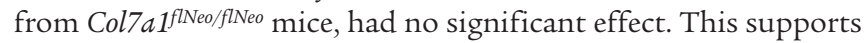
the assumption that the neodeposition of collagen VII at the DEJZ was derived from the injected fibroblasts and that paracrine effects on keratinocytes played a minor role, if any.

The development of an immune response to therapeutic collagen VII or fibroblasts is a putative problem for patients with DEB. Here we observed no collagen VII antibodies in response to the fibroblast injections; both direct and indirect immunofluorescence 
A

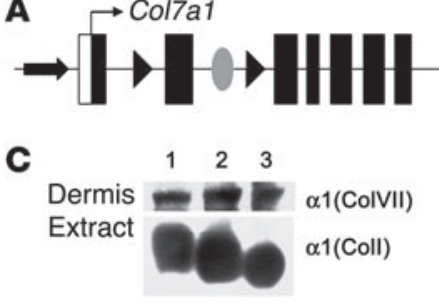

D

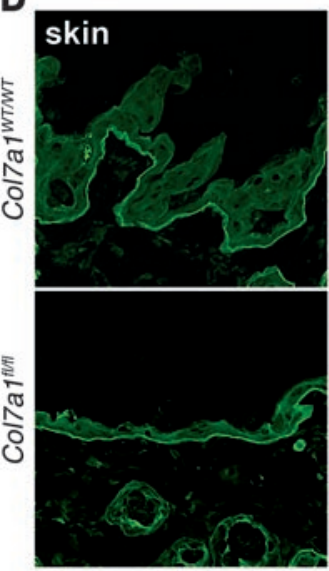

B
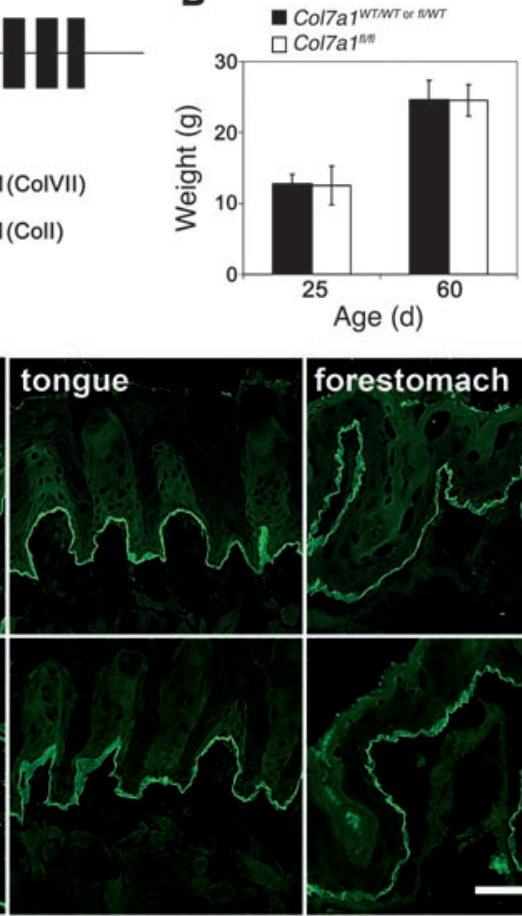

forestomach

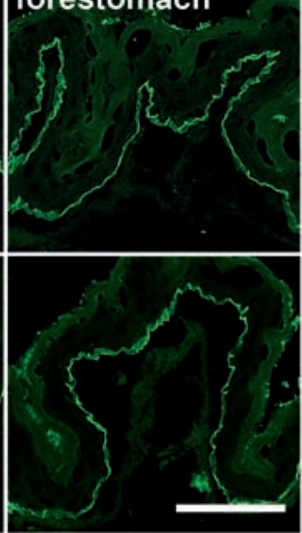

Figure 8

Removal of the PKG-Neo cassette reverts the phenotype

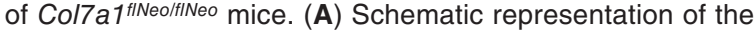
Col7a1 ${ }^{\text {tl }}$ allele. Black boxes denote Col7a1 exons; black triangles denote loxP sites; gray oval denotes Frt site. (B) Col7a $1^{\text {fllft }}$ mice were indistinguishable from their littermates, showing the same weight at 25 and 60 days of age $\left(n=5\right.$ [Col7a $\left.1^{f / / f t}\right] ; 8$ [Col7a1 $\left.{ }^{f / W T}\right]$; 6 [Col7a1 $\left.{ }^{W T / W T}\right]$ ). (C) Immunoblotting of dermal

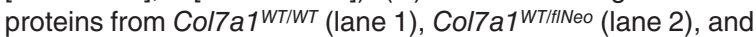
Col7a $1^{\text {fl/fI }}$ (lane 3) mice with the NC2-10 antibody (44) showed no difference in collagen VII expression. Coomassie blue staining of the $\alpha 1$ chain of collagen I is shown as a loading control. (D) Immunofluorescence staining of skin, tongue, and forestomach of Col7a1 $1^{\text {fllfl }}$ mice demonstrated normal collagen VII deposition at the DEJZ. Scale bar: $50 \mu \mathrm{m}$. microscopy of skin and serum of the treated mice remained negative. However, because the hypomorphic mice are inbred and express low levels of collagen VII, an immune response to WT fibroblasts or to collagen VII was not expected. Staining with antibodies to CD11b, a marker for nonspecific inflammation, revealed a moderate inflammatory response in the dermis 7 days after injection. The infiltrate receded within 21 days, but the deposition and function of collagen VII were not impaired. Further studies are needed to clarify whether therapy with cells from different mouse strains, or in patients with DEB who express no or low amounts of collagen VII in the skin, will induce an immune response and make a concomitant immunosuppressive therapy necessary.

Scientists involved in developing biological therapies for genetic diseases have questioned whether complete replacement of the missing gene product is required, or whether partial replacements could be functional. The results of the present study deliver some answers. First, the phenotypic differences between Col7a $1^{\text {flNeo/flNeo }}$ and collagen VII-null mice demonstrate that relatively small alterations in collagen VII levels, $10 \%$ versus null, can strongly influence the stability of the skin. Second, treatment with fibroblast injections demonstrated that elevation of collagen VII levels to approximately $30 \%$ of normal conferred significant resistance of the skin against shearing forces, as shown with the frictional stress test. These observations are in line with previous clinical findings, i.e., heterozygous carriers of COL7A1-null mutations, who have about $50 \%$ of normal collagen VII levels in their skin, are clinically unaffected (37). Thus, if molecular therapies can provide for approximately $30 \%-50 \%$ of normal collagen VII levels, a significant improvement of severe DEB may be achieved, an important step toward developing experimental therapies in humans with DEB.

Treatment of DEB with normal fibroblasts would appear to have major advantages compared with other forms of gene- or cell-based therapies. Fibroblasts are easy to obtain, cultivate, and store, and the delivery of these robust cells via intradermal injections is practical. In view of regulatory and/or gene therapy vector issues, it would be advantageous that the cells synthesize sufficient amounts of the therapeutic protein yet not require genetic modification, because preliminary studies have shown that genetically modified fibroblasts $(29,30)$ may be effective, but can lose the transgene expression within a relatively short time (38).

In summary, we tested cell-based therapy for DEB and showed that intradermal injection of fibroblasts into the hypomorphic mice not only augmented deposition of collagen VII at the DEJZ, but also appeared to improve dermal-epidermal cohesion. These data demonstrate the feasibility of fibroblast-based therapeutic approaches in a preclinical setting and lay a basis for further dissection of quantitative and qualitative details associated with development of a clinically applicable therapy regimen.

\section{Methods}

Generation and maintenance of Col7a fliNeo mice. Transgenic animals were produced as described by Talts et al. (39). To generate the Col7a $1^{\text {flNeo }}$ targeting vector, DNA fragments for homologous recombination and a region containing exon 2 were amplified from tail DNA of 129Sv/CRL mice (accession no. AC174646, sequence of a BAC clone containing the genomic region of murine Col7a1; sequences 164,009-169,109, 157,233-163,520, and 163,323-164,266) using the Expand High Fidelity PCR system (Invitrogen). The BamHI/EcoRI fragment of sequence 164,009-169,109 and a loxP site were introduced upstream of Col7a1 exon 2. PGK-Neo, flanked by 2 Frt sites and 1 loxP site, was inserted downstream of Col7a1 exon 2, followed by an EcoRV/Nsil fragment of sequence 157,233-163,520 (Figure 1). The targeting construct was electroporated into passage-15 R1 mouse ES cells (40). ES cells were selected for recombination with G418, and 384 clones were isolated. For Southern blots, ES cell DNA was digested with BglII and hybridized to a 513-nt external probe (sequence 168,986-169,499). Three positive ES cell clones were expanded and injected into C57BL/6 blastocysts, and 

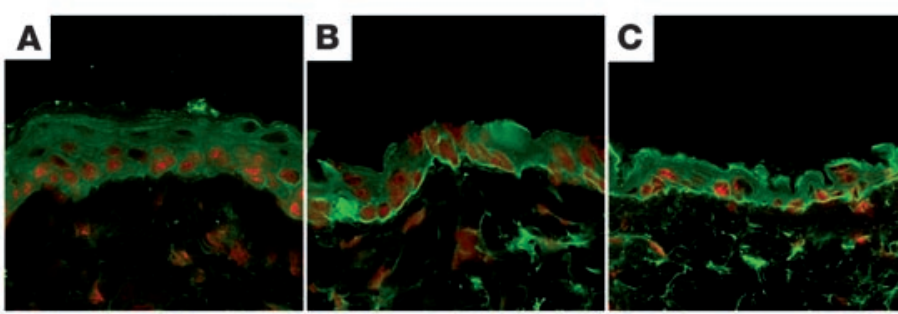

E

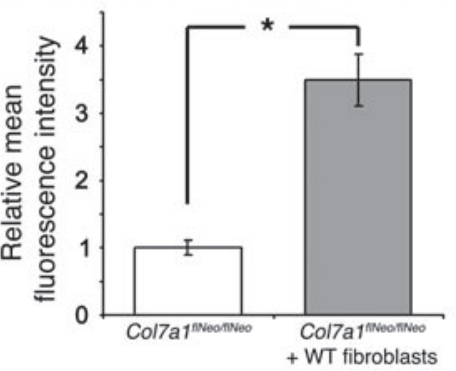

$\mathbf{F}$
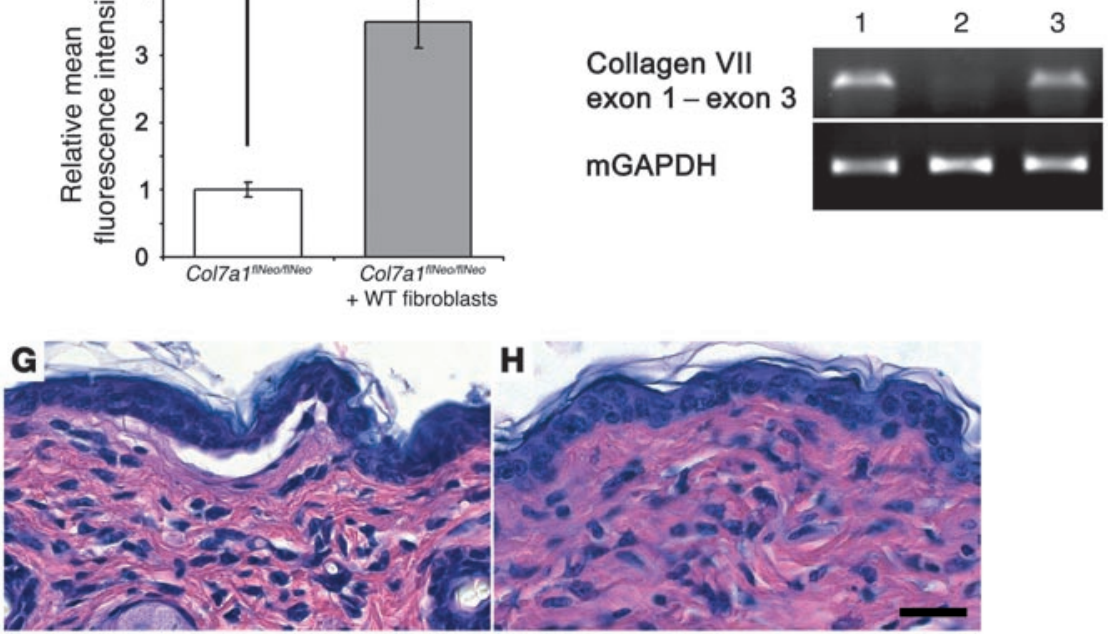

\section{Figure 9}

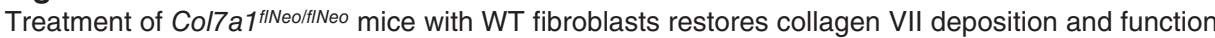
at the DEJZ. (A) In untreated skin of the hypomorph, collagen VII (green) was strongly reduced. (B) Increased collagen VII levels were observed 7 days after 2 intradermal injections of $20 \times 10^{6}$ WT fibroblasts. (C) The increased levels persisted for at least 21 days. Although clearly increased after the treatment, the collagen VII signals remained lower than in Col7a1WT/WT controls (D). Nuclei appear in red. (E) Semiquantitative confocal microscopy ( $n=5$ per group) using identical image settings demonstrated that the relative mean fluorescence intensity of collagen VII fluorescence signals at the DEJZ significantly increased 7 days after treatment of Col7a $1^{\text {IINeo/fINeo }}$ animals with WT fibroblasts compared with untreated skin. ${ }^{*} P<0.01$, Student's $t$ test. $(\mathbf{F})$ Collagen VII mRNA expression in the

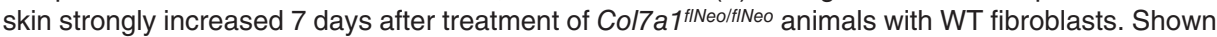
are RT-PCR products of Col7a1 exons 1-3 from Col7a 1WT/WT skin (lane 1), untreated Col7a1 $1^{\text {flNeolfiNeo }}$ skin (lane 2), and Col7a $1^{\text {flNeolfINeo }}$ skin 7 days after treatment (lane 3). GAPDH was used as a control.

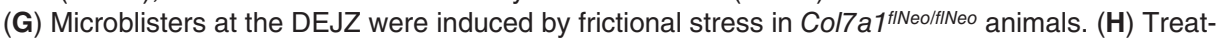
ment with intradermal injection of WT fibroblasts restored the resistance of the skin to shearing forces and abrogated blister formation in response to frictional stress. Scale bars: $25 \mu \mathrm{m}$.

chimeric offspring were mated with C57BL/6CRL females to generate the C57BL/6-TgH(Col7a1flNeo)288LBT transgenic strain. Genotyping was performed by PCR detecting the presence of the loxP site upstream of Col7a1 exon 2 (5'-CGTGGATTCTTCCTAAACTC-3' and 5'-AGGTAGGCGTATTTCTGCCA-3'). The amplification products were 269 and $435 \mathrm{nt}$ for the Col7a $1^{W T}$ and Col7a fliNeo alleles, respectively. The strain was maintained by inbreeding, and Col7a fliNeoflNeo mice were obtained by mating heterozygous siblings. The numbers of animals of the respective genotypes following heterozygous mating were subjected to statistical analysis by $\chi^{2}$ test $\left(\chi^{2}=2.12 ; P=0.34\right)$. Newborn mice were kept with their mothers until weaning at day 28. After we observed the poor condition of the first 15 mice, their diet was changed. Starting on day 10, the pups were also given infant milk cereal (Bebivita) with Nutri-plus Gel dietary supplement (Virbac) ad libitum. This diet continued after weaning instead of solid food. The general condition of the animals was monitored every other day, and 4 clinical parameters were recorded: weight, posture, activity, and fur texture.

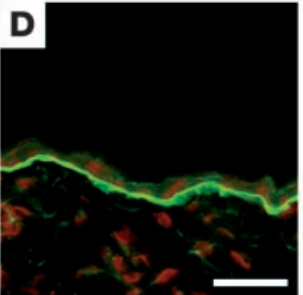

Morphologic analyses. For light microscopic examination, tissue specimens of the back skin, tail, tongue, esophagus, and stomach of Col7a1 flNeo/flNeo mice and control littermates were fixed overnight at $4{ }^{\circ} \mathrm{C}$ in $4 \%$ paraformaldehyde in PBS, dehydrated, and embedded in paraffin. Sections $(3 \mu \mathrm{m})$ were stained with $\mathrm{H} \& \mathrm{E}$ and van Gieson-elastica. For transmission electron microscopy, skin and tongue specimens of newborn mice were fixed in $4 \%$ paraformaldehyde and $2 \%$ glutaraldehyde for at least 2 days at $4^{\circ} \mathrm{C}$, washed twice in $0.1 \mathrm{M}$ cacodylate buffer, and incubated for $1 \mathrm{~h}$ in $1 \%$ osmium tetroxide solution. After dehydration in ethanol $(25 \%, 50 \%, 75 \%$, $90 \%$, and $100 \%$ ) and propylene oxide, samples were embedded in an epoxide resin. Sections of 70-nm thickness were mounted on microscopy grids and stained with 5\% uranyl acetate and Reynold's solution.

Immunofluorescence analyses and semiquantitative confocal microscopy. For indirect immunofluorescence staining of the skin, $4-\mu \mathrm{m}$ cryosections were fixed for 10 $\mathrm{min}$ in acetone $\left(-20^{\circ} \mathrm{C}\right)$ and air dried. The sections were blocked with $2 \%$ BSA for 30 min, incubated overnight at $4^{\circ} \mathrm{C}$ with the primary antibodies followed by $1 \mathrm{~h}$ with the secondary antibodies, and mounted in Mowiol after intensive washing. The following primary antibodies were used: polyclonal anti-collagen VII (Calbiochem), monoclonal anti- $\alpha_{6}$ integrin (Progen), anti- $\alpha$-SMA (Sigma-Aldrich), anti-tenascin C (R\&D Systems), anti-CD11b (BD Biosciences - Pharmingen), anti-TGF- $\beta 1$ (25 kDa; Promega), and anti-CTGF (Abcam). The secondary antibodies were Alexa Fluor 488-conjugated anti-rabbit (Invitrogen), FITC-conjugated anti-rat (Abcam), and Cy5-conjugated streptavidin (BD Biosciences - Pharmingen). Nuclei were stained with DAPI (Sigma-Aldrich).

For detection of autoantibodies by direct immunofluorescence, cryosections of Col7 a $1^{\text {flNeofflNeo }}$ mouse skin excised 21 days after treatment were incubated with biotinylated anti-mouse IgG (Axxora) and streptavidin-Alexa Fluor 488 (Invitrogen). The skin of untreated Col7a $1^{W T / W T}$ mice was used as a control. For analysis of circulating autoantibodies by indirect immunofluorescence, cryosections of Col7a1 $1^{W T / W T}$ mouse skin were incubated with sera of treated

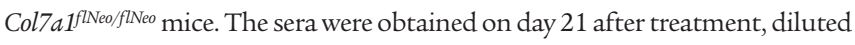
1:10, and incubated and detected as described above.

Confocal microscopy-based semiquantitative comparison of the intensity of collagen VII immunofluorescence signals at the DEJZ in mice with or without fibroblast treatment was performed as described previously (41). A total of 5 cryosections per group was stained for collagen VII. Stacks with 5 sections ( $z$ distance, $0.8 \mu \mathrm{m}$ ) of monochrome 12-bit gray-level images were recorded with a Zeiss LSM510 confocal microscope equipped with a LD LCIApochromat $\times 25$, 0.8 glycerine objective (Carl Zeiss MicroImaging) using identical settings and multichannel acquisition after excitation at $405 \mathrm{~nm}$ 
(DAPI; emission BP, 420-480 nm) and $488 \mathrm{~nm}$ (Alexa 488; emission LP, 505 $\mathrm{nm}$ ). The pinhole was adjusted to 1 airy unit (corresponding to an optical slice thickness of $1.6 \mu \mathrm{m})$. Images with $2,048 \times 2,048$ pixels $(0.18 \mu \mathrm{m}$ isotropic) were recorded. The image plane with the highest mean intensity signal was chosen for analysis. Data analysis was performed with the full version of the Zeiss LSM software (version 4.2). The mean intensity of the collagen VII signal at the DEJZ was obtained by measuring 3 independent DEJZ areas (defined as $n=1$ ) and subtracting the intensity of an adjacent area of the same size as background. Relative intensities were calculated based on the mean collagen VII intensity at the DEJZ of untreated Col7 a flNeo/flNeo mice. $^{\text {. }}$

Cultivation of murine keratinocytes and fibroblasts. Keratinocytes and fibroblasts were isolated from the skin of Col7a $1^{\text {flNeofflNeo mice and littermates }}$ essentially as described previously $(42,43)$. Briefly, the skin was washed with $70 \%$ ethanol, and epidermis and dermis were separated by digestion with $0.25 \%$ trypsin $(\mathrm{w} / \mathrm{v})$ for $30 \mathrm{~min}$ at $37^{\circ} \mathrm{C}$. After stopping the reaction with PBS containing $10 \% \mathrm{FCS}$, the epidermis was removed and incubated for $30 \mathrm{~min}$ at $37^{\circ} \mathrm{C}$ under vigorous shaking to separate the keratinocytes. After passing the keratinocytes through a $70-\mu \mathrm{m}$ sieve, $10^{5} \mathrm{cells} / \mathrm{cm}^{2}$ were plated in serum-free keratinocyte growth medium supplemented with 100 $\mathrm{U} / \mathrm{ml}$ penicillin, $100 \mu \mathrm{g} / \mathrm{ml}$ streptomycin, and $0.25 \mu \mathrm{g} / \mathrm{ml}$ amphotericin $\mathrm{B}$ (all from Invitrogen). For isolation of fibroblasts after removal of the epidermis, the dermis was digested with $415 \mu \mathrm{g} / \mathrm{ml}$ collagenase from Clostridium histolyticum (Sigma-Aldrich) for $30 \mathrm{~min}$ at room temperature, and $10^{5} \mathrm{cells} / \mathrm{cm}^{2}$ were plated in a DMEM/Ham's F12-mixture (1:1; Invitrogen) containing $10 \%$ FCS and antibiotics. The cells were maintained at $37^{\circ} \mathrm{C}$, $5 \% \mathrm{CO}_{2}$, and $95 \%$ humidity, and medium was replaced every $48 \mathrm{~h}$.

Spontaneous immortalization of murine keratinocytes. Primary murine keratinocytes were grown in serum-free keratinocyte growth medium and passaged every 7-10 days until cultures became senescent. After 4-6 weeks, single keratinocytes regained proliferative potential and clones were expanded. From each genotype, 2 cell lines were established. The cells were plated onto coverslips and immunostained for the expression of cytokeratins. Genotyping of the spontaneously immortalized keratinocytes confirmed the Col7a1 status of these cell lines.

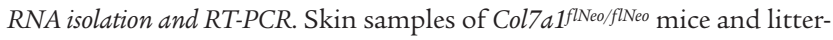
mates were frozen and homogenized in liquid $\mathrm{N}_{2}$ with mortar and pestle. Total RNA was isolated using TRIzoL (Amersham) or the RNeasy Fibrous Tissue Mini Kit (Qiagen) according to the manufacturer's protocol. Keratinocytes and fibroblasts were grown to $90 \%$ confluence, and RNA was isolated using the QIAamp RNA Blood Mini Kit (Qiagen). RNA (500 ng) was reverse transcribed (Advantage RT-for-PCR Kit; BD Biosciences), and collagen VII mRNA was amplified with the following primers: Col7a1 exon 1exon 2, 5'-CTGCAGAGATCCTGATGGGA-3' and 5'-GATCATCACTGTACTGCACT-3'; Col7a1 exon 2-exon 3, 5'-ATGCTGCTGACATCGTGTTC-3' and 5'-CAGGACGTGTTAGACGAGGC-3' (Taq DNA Polymerase; Invitrogen). We performed 3 '-RACE using 5'-GACTCGAGTCGACATCGATTTTTTTTTTTTTTTTT-3' for reverse transcription (Advantage RT-forPCR Kit; BD Biosciences), followed by amplification of PGK-Neo containing $3^{\prime}$ ends with 5'-GAGCGAGCACGTACTCGGAT-3' and 5'-GACTCGAGTCGACATCG-3' . Amplification of murine GAPDH demonstrated equal cDNA amounts in all samples. Semiquantitative densitometry was performed with Gel-Pro Express software version 4.0 (Media Cybernetics Inc.).

Cell and tissue extracts and Western blotting. Epidermis and dermis were separated by incubation in a buffer containing $1 \mathrm{M} \mathrm{NaCl}, 0.05 \mathrm{M}$ Tris/ $\mathrm{HCl}$ (pH 7.4), $2 \mathrm{mM}$ EDTA, and $1 \mathrm{mM}$ Pefabloc (Merck) at $4^{\circ} \mathrm{C}$ for $4-5 \mathrm{~h}$. After removal of the epidermis, the dermis was placed upside-down in a buffer containing $0.8 \mathrm{M}$ urea, $0.1 \mathrm{M}$ Tris/ $\mathrm{HCl}$ ( $\mathrm{pH} 6.8$ ), $2 \% \mathrm{SDS}, 5 \%$ glycerol, $1 \mathrm{mM}$ DTT, and bromophenol blue, and dermal proteins were extracted for $5 \mathrm{~min}$ at $95^{\circ} \mathrm{C}(6,34,44)$. Subconfluent keratinocytes and fibroblasts were cultured for $48 \mathrm{~h}$ in the presence of $50 \mu \mathrm{g} / \mathrm{ml}$ ascorbate to allow for full pro- lyl and lysyl hydroxylation of newly synthesized collagens. The cells were washed with ice-cold PBS and extracted for $30 \mathrm{~min}$ on ice with a lysis buffer containing $1 \%$ Nonidet P-40, $0.1 \mathrm{M} \mathrm{NaCl}, 0.025 \mathrm{M}$ Tris/ $\mathrm{HCl}$ ( $\mathrm{pH} 7.4$ ), $10 \mathrm{mM}$ EDTA, and the proteinase inhibition cocktail set III (Calbiochem). The extracts were clarified by centrifugation at $14,000 \mathrm{~g}$ for $15 \mathrm{~min}$ at $4^{\circ} \mathrm{C}$. Proteins were separated on 7\% SDS-PAGE, and collagen VII expression was assayed by immunoblotting with the NC2-10 antibody (44), followed by densitometry. To ensure equal loading of the samples, gels of dermis extracts were stained with Coomassie blue (BioRad), and cell extracts were immunoblotted with an anti- $\beta$-tubulin antibody (Abcam).

$X$-ray imaging. High-resolution digital radiographies of mouse extremities were obtained in 2 planes with an Agfa ADM 1000 radiography unit (Agfa) with the following parameters: $32 \mathrm{kV} ; 25 \mathrm{mAs}$; image size, $24 \times 29 \mathrm{~cm}$; matrix, 3,328 $\times 4,096$ pixels. This resulted in nearly isotropic resolution, with pixel size of $71 \times 72 \mu \mathrm{m}$. Window settings were optimized manually.

High-resolution MRI. MRI was performed on a 9.4 T Bruker animal system (Bruker Biospin 94/20; Bruker Biospin) equipped with a BGS12 gradient system capable of $400 \mathrm{mT} / \mathrm{m}$ gradient strength and $6,000 \mathrm{mT} / \mathrm{m} / \mathrm{s}$ slew rate. A quadratur coil with $35-\mathrm{cm}$ inner diameter was used for radio frequency excitation and data acquisition. Whole-body mouse images were acquired in 3 stages with 54 slices each using a fat-suppressed T1- and T2-weighted rapid acquisition with relaxation enhancement (RARE) sequence. These 162 slices had a thickness of $500 \mu \mathrm{m}$ and an in-plane resolution of $130 \mu \mathrm{m}$. Parameters for T1-weighted imaging included repetition time (TR) of 1,500 $\mathrm{ms}$, echo time (TE) of $7.5 \mathrm{~ms}$, and turbo factor 4 . The T2-weighted images were acquired using TR of 7,500 ms, TE of $40 \mathrm{~ms}$, and turbo factor 8 . In addition, images from limbs were acquired separately using a high-resolution 3D T1-weighted Gradient Echo sequence with $\alpha$ of $20^{\circ}$, TR of $13.4 \mathrm{~ms}$, and TE of $2.9 \mathrm{~ms}$ (asymmetric echo). Resolution was about $100 \mu \mathrm{m}$ isotropic.

Hematological analysis. Peripheral blood from adult (9-12 weeks old) mice was collected, and the number of red and white blood cells was counted using a hemocytometer. Cell numbers, cell size, and hematocrit and hemoglobin levels were determined with the hematology analyzer Sysmex XT1800i. White blood cells were identified by staining with FITC-labeled monoclonal antibodies RA3-6B2 anti-B220, 145-2C11 anti-CD3, and RB6$8 \mathrm{C} 5$ anti-Gr-1 (all BD Biosciences - Pharmingen) and quantified using a FACSCalibur flow cytometer (BD Biosciences).

Cell therapy with fibroblastinjection. Forcell therapy of 5-week-old Col7 a f fliNeofflNeo mice $(n=19)$, fibroblasts from Col7a $1^{W T / W T}$ mice $(n=16)$ or Col7a $1^{\text {flNeofflNeo }}$ mice $(n=3)$ in passage $2-3$ were trypsinized, extensively washed with $0.9 \%$ $\mathrm{NaCl}$, and adjusted to a final concentration of $40 \times 10^{6} \mathrm{cells} / \mathrm{ml}$. Mice were anesthetized i.p. with ketamine $(60 \mathrm{mg} / \mathrm{kg})$ and medetomedine $(0.25 \mathrm{mg} / \mathrm{kg})$, and the dorsal skin was shaved and disinfected with Octenisept (Schülke $\&$ Mayr). Into a defined area of $2 \times 3 \mathrm{~cm}, 20 \times 10^{6}$ cells were injected intradermally with a 27 -gauge needle. The procedure was repeated after 7 days, and mice were sacrificed 7, 14, and 21 days after the second injection. Prior to dissection, the mice were anesthetized with isoflurane inhalation, and treated and untreated dorsal skin areas of $1 \mathrm{~cm}^{2}$ were marked with a pen and exposed to a mechanical rubber stress test. The skin was gently stretched, and mechanical shearing forces were applied by the same investigator by repeated (20 times), intense, unidirectional rubbing with a pencil eraser. After $60 \mathrm{~min}$, skin specimens from treated and untreated areas were excised and processed for RNA isolation as well as histopathological and indirect immunofluorescence analysis.

Approval of animal experiments and studies on humans. The animal experiments included in this study were approved by the state review board (Regierungspräsidium Freiburg, Freiburg, Germany; approval no. 35/9185.81/G-05/08 and 35/9185.81/G-06/79). Studies on DEB patients were approved by the ethical committee of the University of Freiburg (approval no. 16603). Patients gave informed consent for the use of their 
material and clinical data for research and publication; all clinical investigations were conducted according to the Declaration of Helsinki.

Statistics. Significance of differences in animal weight was determined by 2 -tailed Student's $t$ test. Mendelian inheritance was confirmed by $\chi^{2}$ test, and fluorescence intensities obtained by quantification of confocal microscopy images were compared using 2-tailed Student's $t$ test. A $P$ value less than 0.01 was considered significant.

\section{Acknowledgments}

The authors thankfully acknowledge the expert technical assistance of Claudia Ehret, Käthe Thoma, Ursula Voith, Margit Schubert, and Anne-Katrin Becker. They thank Eva Mangel and the team of the BioMed Center of the University Medical Center Freiburg for excellent animal care, Dietmar Geiger for help with X-ray imaging, Roland Nitschke for help with confocal microscopy, and Marcel Müller for help with statistical analysis. This work was supported in part by grant Br1475/8-2 from the Deutsche Forsc- hungsgemeinschaft, EU STREP grant "Skintherapy," and a grant from DEBRA Europe to L. Bruckner-Tuderman; by the Network Epidermolysis Bullosa, Projects 8 and 9, from the Federal Ministry for Education and Research (BMBF) to L. Bruckner-Tuderman and R. Fässler; by the Max Planck Society to R. Fässler; and by a grant from the German Dermatologic Society (DDG) to J.S. Kern.

Received for publication October 22, 2007, and accepted in revised form January 30, 2008.

Address correspondence to: Leena Bruckner-Tuderman, Department of Dermatology, University Medical Center Freiburg, Hauptstr. 7, 79104 Freiburg, Germany. Phone: 49-671-270-6716; Fax: 49671-270-6936; E-mail: bruckner-tuderman@uniklinik-freiburg.de.

Anja Fritsch, Stefan Loeckermann, and Johannes S. Kern contributed equally to this work.
1. Has, C., and Bruckner-Tuderman, L. 2006. Molecular and diagnostic aspects of genetic skin fragility. J. Dermatol. Sci. 44:129-144.

2. Ryynanen, J., et al. 1992. Type VII collagen gene expression by cultured human cells and in fetal skin. Abundant mRNA and protein levels in epidermal keratinocytes. J. Clin. Invest. 89:163-168.

3. Rattenholl, A., et al. 2002. Proteinases of the bone morphogenetic protein-1 family convert procollagen VII to mature anchoring fibril collagen. J. Biol. Chem. 277:26372-26378.

4. Aumailley, M., Has, C., Tunggal, L., and BrucknerTuderman, L. 2006. Molecular basis of inherited skin-blistering disorders, and therapeutic implications. Expert Rev. Mol. Med. 8:1-21.

5. Fine, J.D., McGrath, J., and Eady, R.A. 2000. Inherited epidermolysis bullosa comes into the new millenium: a revised classification system based on current knowledge of pathogenetic mechanisms and the clinical, laboratory, and epidemiologic findings of large, well-defined patient cohorts. J. Am. Acad. Dermatol. 43:135-137.

6. Kern, J.S., Kohlhase, J., Bruckner-Tuderman, L., and Has, C. 2006. Expanding the COL7A1 mutation database: novel and recurrent mutations and unusual genotype-phenotype constellations in 41 patients with dystrophic epidermolysis bullosa. J. Invest. Dermatol. 126:1006-1012.

7. Varki, R., Sadowski, S., Uitto, J., and Pfendner, E. 2007. Epidermolysis bullosa. II. Type VII collagen mutations and phenotype-genotype correlations in the dystrophic subtypes. J. Med. Genet. 44:181-192.

8. Bhowmick, N.A., et al. 2004. TGF-beta signaling in fibroblasts modulates the oncogenic potential of adjacent epithelia. Science. 303:848-851.

9. Heinonen, S., et al. 1999. Targeted inactivation of the type VII collagen gene (Col7a1) in mice results in severe blistering phenotype: a model for recessive dystrophic epidermolysis bullosa. J. Cell Sci. 112:3641-3648.

10. Cao, T., Longley, M.A., Wang, X.J., and Roop, D.R. 2001. An inducible mouse model for epidermolysis bullosa simplex: implications for gene therapy. J. Cell Biol. 152:651-656.

11. Rodgers, K.D., Sasaki, T., Aszodi, A., and Jacenko, O. 2007. Reduced perlecan in mice results in chondrodysplasia resembling Schwartz-Jampel syndrome. Hum. Mol. Genet. 16:515-528.

12. Vermot, J., Niederreither, K., Garnier, J.M., Chambon, P., and Dolle, P. 2003. Decreased embryonic retinoic acid synthesis results in a DiGeorge syndrome phenotype in newborn mice. Proc. Natl. Acad. Sci.U. S. A. 100:1763-1768.

13. Lupher, M.L., Jr., and Gallatin, W.M. 2006. Regulation of fibrosis by the immune system. Adv. Immu- nol. 89:245-288.

14. Hinz, B. 2007. Formation and function of the myofibroblast during tissue repair. J. Invest. Dermatol. 127:526-537.

15. Chiquet-Ehrismann, R., and Chiquet, M. 2003. Tenascins: regulation and putative functions during pathological stress. J. Pathol. 200:488-499.

16. Martin, P., and Leibovich, S.J. 2005. Inflammatory cells during wound repair: the good, the bad and the ugly. Trends Cell Biol. 15:599-607.

17. Werner, S., Krieg, T., and Smola, H. 2007. Keratinocyte-fibroblast interactions in wound healing. J. Invest. Dermatol. 127:998-1008.

18. Hinz, B., et al. 2007. The myofibroblast. One function, multiple origins. Am. J. Pathol. 170:1807-1816.

19. Gabbiani, G. 2003. The myofibroblast in wound healing and fibrocontractive diseases. J. Pathol. 200:500-503.

20. Ingber, D.E. 2002. Cancer as a disease of epithelialmesenchymal interactions and extracellular matrix regulation. Differentiation. 70:547-560.

21. Coussens, L.M., and Werb, Z. 2002. Inflammation and cancer. Nature. 420:860-867.

22. Maeda, H., and Akaike, T. 1998. Nitric oxide and oxygen radicals in infection, inflammation, and cancer. Biochemistry Mosc. 63:854-865.

23. Yamanishi, Y., et al. 2002. Regional analysis of p53 mutations in rheumatoid arthritis synovium. Proc. Natl. Acad. Sci. U. S. A. 99:10025-10030.

24. Hayashi, N., and Cunha, G.R. 1991. Mesenchymeinduced changes in the neoplastic characteristics of the Dunning prostatic adenocarcinoma. Cancer Res. 51:4924-4930.

25. Cooper, M., and Pinkus, H. 1977. Intrauterine transplantation of rat basal cell carcinoma as a model for reconversion of malignant to benign growth. Cancer Res. 37:2544-2552.

26. Gache, Y., et al. 2004. Construction of skin equivalents for gene therapy of recessive dystrophic epidermolysis bullosa. Hum. Gene Ther. 15:921-933.

27. Ortiz-Urda, S., et al. 2002. Stable nonviral genetic correction of inherited human skin disease. Nat. Med. 8:1166-1170.

28. Woodley, D.T., et al. 2004. Intradermal injection of lentiviral vectors corrects regenerated human dystrophic epidermolysis bullosa skin tissue in vivo. Mol. Ther. 10:318-326.

29. Woodley, D.T., et al. 2003. Normal and gene-corrected dystrophic epidermolysis bullosa fibroblasts alone can produce type VII collagen at the basement membrane zone. J. Invest. Dermatol. 121:1021-1028.

30. Ortiz-Urda, S., et al. 2003. Injection of genetically engineered fibroblasts corrects regenerated human epidermolysis bullosa skin tissue. J. Clin. Invest. 111:251-255.
31. Woodley, D.T., et al. 2004. Injection of recombinant human type VII collagen restores collagen function in dystrophic epidermolysis bullosa. Nat. Med. 10:693-695.

32. Sitaru, C., et al. 2005. Induction of dermal-epidermal separation in mice by passive transfer of antibodies specific to type VII collagen. J. Clin. Invest. 115:870-878

33. Woodley, D.T., et al. 2005. Evidence that anti-type VII collagen antibodies are pathogenic and responsible for the clinical, histological, and immunological features of epidermolysis bullosa acquisita. J. Invest. Dermatol. 124:958-964.

34. Bruckner-Tuderman, L., Guscetti, F., and Ehrensperger, F. 1991. Animal model for dermolytic mechanobullous disease: sheep with recessive dystrophic epidermolysis bullosa lack collagen VII. J. Invest. Dermatol. 96:452-458.

35. Baldeschi, C., et al. 2003. Genetic correction of canine dystrophic epidermolysis bullosa mediated by retroviral vectors. Hum. Mol. Genet. 12:1897-1905.

36. Wong, T., et al. 2007. A single intradermal injection of allogeneic fibroblasts can increase type VII collagen expression in recessive dystrophic epidermolysis bullosa for at least 3 months [supplement]. J. Invest. Dermatol. 127:S91.

37. Hilal, L., et al. 1993. A homozygous insertion-deletion in the type VII collagen gene (COL7A1) in Hallopeau-Siemens dystrophic epidermolysis bullosa. Nat. Genet. 5:287-293.

38. Krueger, G.G., Morgan, J.R., and Petersen, M.J. 1999. Biologic aspects of expression of stably integrated transgenes in cells of the skin in vitro and in vivo. Proc. Assoc. Am. Physicians. 111:198-205.

39. Talts, J.F., Brakebusch, C., and Fassler, R. 1999. Integrin gene targeting. Methods Mol. Biol. 129:153-187.

40. Nagy, A., Rossant, J., Nagy, R., Abramow-Newerly, W., and Roder, J.C. 1993. Derivation of completely cell culture-derived mice from early-passage embryonic stem cells. Proc. Natl. Acad. Sci. U. S. A. 90:8424-8428.

41. Zaha, V., et al. 2005. Discrepancy between GLUT4 translocation and glucose uptake after ischemia. Mol. Cell. Biochem. 278:129-137.

42. Caldelari, R., Suter, M.M., Baumann, D., De Bruin, A., and Muller, E. 2000. Long-term culture of murine epidermal keratinocytes. J. Invest. Dermatol. 114:1064-1065.

43. Echtermeyer, F., et al. 2001. Delayed wound repair and impaired angiogenesis in mice lacking syndecan-4. J. Clin. Invest. 107:R9-R14.

44. Bruckner-Tuderman, L., et al. 1995. Immunohistochemical and mutation analyses demonstrate that procollagen VII is processed to collagen VII through removal of the NC-2 domain. J. Cell Biol. 131:551-559. 